Tohoku J. exp. Med., 1985, 146, 27-32

\title{
Immunohistochemical Localization of Carcinoembryonic Antigen in Adenocarcinoma of the Endocervix and the Endometrium
}

\author{
Toshiniko Toki, Naohiro Oikawa, Toru Tase, Yuichi \\ Wada, Akira Yajima and Masakuni Suzuki \\ Department of Obstetrics and Gynecology, Tohoku \\ University School of Medicine, Sendai 980
}

\begin{abstract}
Toki, T., Oikawa, N., Tase, T., Wada, Y., Yajima, A. and Suzuki, M. Immunohistochemical Localization of Carcinoembryonic Antigen in Adenocarcinoma of the Endocervix and the Endometrium. Tohoku J. exp. Med., 1985, 146 (1), 27-32 Immunoperoxidase localization of carcinoembryonic antigen (CEA) was performed on tissue sections of adenocarcinoma of endocervical and endometrial origin to clarify the criteria for their differentiation. Twenty-two $(96 \%)$ of 23 endocervical and $32(70 \%)$ of 46 endometrial adenocarcinomas revealed positive CEA immunostain. The extent of staining did not seem to correlate with the degree of differentiation in either kind of cancer. Among the positive cases, CEA staining was located over whole cytoplasm in $18(80 \%)$ of 22 positive endocervical adenocarcinomas and on apical surface in $24(75 \%)$ of 32 positive endometrial adenocarcinomas. The pattern of CEA distribution in endocervical adenocarcinoma of endometrioid type was not similar to that observed for primary endometrial adenocarcinoma.__ immunohistochemistry ; carcinoembryonic antigen; endocervical adenocarcinoma; endometrial adenocarcinoma
\end{abstract}

Carcinoembryonic antigen (CEA) first described by Gold and Freedman (1965) has now become one of the best known tumor markers. Although CEA was originally described as a tumor associated antigen of the colon, it is demonstrated in serum of patients with various kinds of neoplasms.

In patients with gynecological malignancies, CEA is demonstrated in serum by radioimmunoassay and in tissue by immunohistochemistry. Wahlström and colleagues (1979) suggested that the possibility of presence of CEA is much higher in an adenocarcinoma of endocervical origin as compared with that of endometrial origin and that this might be an important consideration in making differential diagnosis. On the other hand, CEA has also been detected in some cases of endometrial adenocarcinoma by several other authors (Goldenberg et al. 1978; Cohen et al. 1982 ; Ueda et al. 1983; Okudaira et al.1984).

The aim of the present study is to clarify the difference in localization of CEA

Received November 12, 1984 ; accepted for publication November 30, 1984. 
between endocervical and endometrial adenocarcinomas and to distinguish these two types of uterine cancer.

\section{Materials and Methods}

The materials consisted of 23 cases of endocervical and 46 cases of endometrial adenocarcinomas. All cases were collected from the specimens obtained by hysterectomy performed at the Department of Obstetrics and Gynecology, Tohoku University Hospital and National Sendai Hospital (kindly provided by Dr. Tsuneo Namiki). The primary site of carcinoma was clinically and histologically defined in all cases.

All slices were recut from routine formalin-fixed and paraffin-embedded blocks ; these materials were obtained during the period from 1981 to 1984 . Three micron serial sections were deparaffinized and stained with hematoxylin-eosin and alcian blue at $\mathrm{pH}$ 2.5.

Immunoperoxidase for CEA. Immunohistochemical study was performed by the $\mathrm{ABC}$ method (Hsu et al. 1981) using Vectastain rabbit IgG kit (Vector Laboratories). Anti-CEA rabbit antiserum (Dakopatts) was absorbed with perchloric acid extract of normal human spleen tissue for $12 \mathrm{hr}$ at $4^{\circ} \mathrm{C}$ before use to remove reactivity with non-specific cross-reacting antigen (Wagener et al. 1978). Endogenous peroxidase activity was blocked with $0.3 \%$ hydrogen peroxide in anhydrous methanol. Tissue sections were then rinsed and incubated for 20 min at $37^{\circ} \mathrm{C}$ in $0.1 \%$ trypsin $1: 250$ (Difco) in $0.05 \mathrm{M}$ Tris- $\mathrm{HCl}$ buffer $\mathrm{pH} 7.6$ with the addition of $0.1 \% \mathrm{CaCl}_{2}$ (Hautzer et al. 1980). The sections were rinsed with phosphatebuffered saline (PBS), and then treated sequentially with normal goat serum $(1: 20,15 \mathrm{~min})$, anti-CEA (1:200, $30 \mathrm{~min})$, biotinylated anti-rabbit $\operatorname{IgG}(1: 200,30 \mathrm{~min})$ and Avidin-Biotin peroxidase Complex (1:100,30 min). Each reaction was carried at room temperature in a moist chamber, with intervening cold PBS washes. The bound peroxidase was visualized by immersing the slices for 3-4 min at room temperature in a solution of $0.02 \%$ diaminobenzidine tetrahydrochloride (Dotite) and $0.005 \%$ hydrogen peroxide containing $10 \mathrm{mM}$ sodium azide. The nuclei were counterstained with methyl green and the slices were dehydrated and mounted. The primary antiserum was replaced with normal rabbit serum $1: 20$ as a negative control.

Evaluation of the staining. If any small portion of the cancer tissue showed a positive staining, the tissue was labelled as weakly positive or "plus", whereas if more than $50 \%$ of the cancer cells showed a positive staining, the sample was labelled as strongly positive or "plus plus".

\section{RESUlts}

The results of CEA localization of 23 cases of endocervical and 46 cases of endometrial adenocarcinomas are summarized in Table 1 and illustrated in Figs. $1,2,3,4$ and 5 . Twenty-two cases $(96 \%)$ of 23 endocervical adenocarcinomas

TABLE 1. CEA staining in adenocarcinoma of the uterus

\begin{tabular}{lccc}
\hline & $(-)$ & $\begin{array}{c}\text { CEA } \\
(+)\end{array}$ & $($ H $)$ \\
\hline $\begin{array}{c}\text { Endocervical } \\
\text { adenocarcinoma }\end{array}$ & 1 & 11 & 11 \\
$\begin{array}{c}\text { Endometrial } \\
\text { adenocarcinoma }\end{array}$ & 14 & 31 & 1 \\
\hline
\end{tabular}




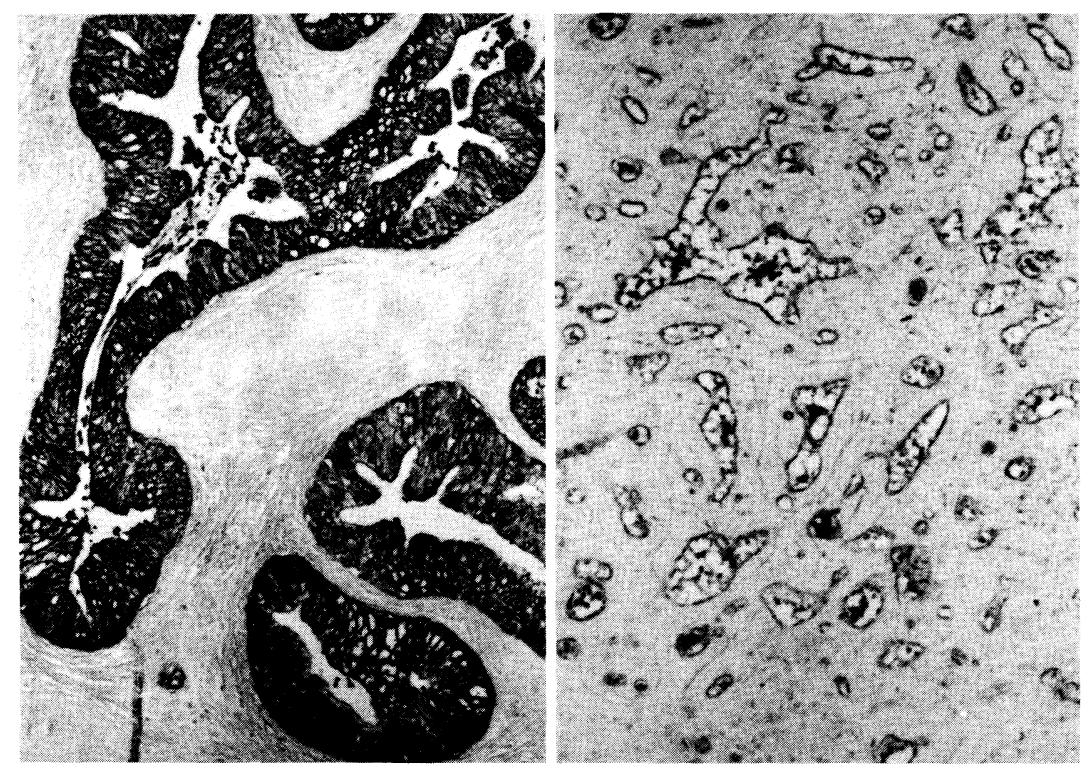

Fig. 1 (left). Endocervical adenocarcinoma with diffuse positive staining for CEA over whole cytoplasm $(\times 100)$.

Fig. 2 (right). Endocervical adenocarcinoma with positive staining for CEA on apical surface and luminal secretion $(\times 100)$.
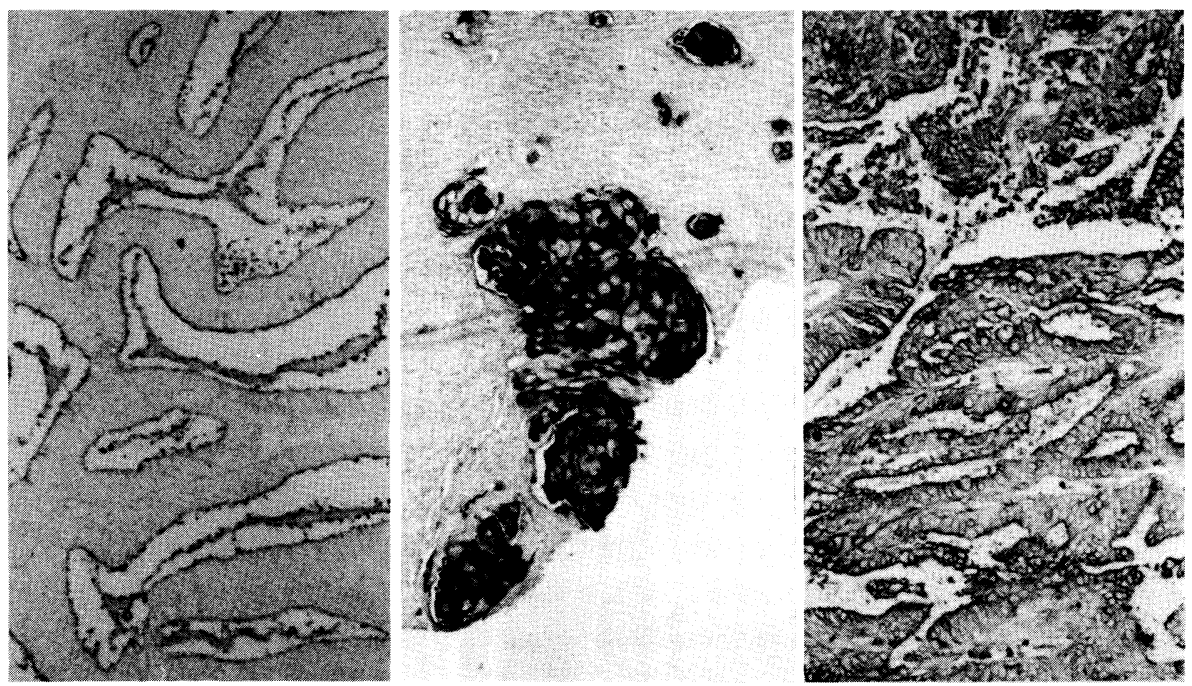

Fig. 3 (left). Endometrial adenocarcinoma showing positive staining for CEA on apical surface $(\times 90)$.

Fig. 4 (middle). Endometrial adenosquamous carcinoma showing positive staining for CEA on cytoplasm of the squamous portion $(\times 90)$.

Fig. 5 (right). Endometrial adenocarcinoma showing diffuse positive staining for CEA over whole cytoplasm $(\times 90)$. 
TABle 2. Degree of differentiation and CEA immunostain in adenocarcinoma of the uterus

\begin{tabular}{ccccccc}
\hline & $\begin{array}{c}\text { Endocervical } \\
\text { adenocarcinoma }(\mathrm{N}=23)\end{array}$ & \multicolumn{4}{c}{$\begin{array}{c}\text { Endometrial } \\
\text { adenocarcinoma }(\mathrm{N}=46)\end{array}$} \\
\cline { 2 - 7 } & $(-)$ & $\begin{array}{c}\text { CEA } \\
(+)\end{array}$ & $(+)$ & $(-)$ & $\begin{array}{c}\text { CEA } \\
(+)\end{array}$ & $(+)$ \\
\hline G1 & 1 & 6 & 8 & 10 & 21 & 0 \\
G2 & 0 & 5 & 1 & 2 & 7 & 1 \\
G3 & 0 & 0 & 2 & 2 & 3 & 0 \\
\hline
\end{tabular}

G1, well differentiated; G2, moderately differentiated; G3, poorly differentiated.

TABLE 3. Distribution of positive CEA immunostain in adenocarcinoma of the uterus

\begin{tabular}{cccc}
\hline & $\begin{array}{c}\text { Whole } \\
\text { cytoplasm }\end{array}$ & $\begin{array}{c}\text { Apical } \\
\text { surface }\end{array}$ & $\begin{array}{c}\text { Squamous } \\
\text { portion }\end{array}$ \\
\hline $\begin{array}{c}\text { Endocervical } \\
\text { adenocarcinoma }\end{array}$ & 18 & 4 & 0 \\
Endocervical type & 11 & 2 & \\
Endometrioid type & 6 & 2 & 7 \\
Endometrial & 8 & 24 & \\
adenocarcinoma & & & \\
\hline
\end{tabular}

revealed positive CEA immunostain. One negative case was clear cell adenocarcinoma. Among 22 positive cases, 11 were weakly positive and other 11 were strongly positive.

Thirty-two cases $(70 \%)$ of 46 endometrial adenocarcinomas showed positive CEA immunostain. Among the positive cases, 31 were weakly positive with only one case labelled as strongly positive.

Table 2 shows the degree of tumor differentiation and the extent of CEA staining. No correlation was noted for either endocervical or endometrial adenocarcinomas. Moreover, comparison of CEA staining with alcian blue staining revealed no correlation for either type of uterine cancer.

Table 3 shows the distribution of positive CEA immunostain in endocervical and endometrial adenocarcinomas. CEA was present on whole cytoplasm in 18 cases $(80 \%)$ of 22 positive endocervical adenocarcinomas (Fig. 1 ). In 4 cases, CEA was focally stained on apical surface (Fig. 2). In two cases of cervical adenocarcinoma with squamous component, CEA was negative in the squamous portion.

In contrast, CEA was focally stained on apical surface or apical surface plus squamous portion in 24 cases $(75 \%)$ of 32 positive endometrial adenocarcinomas (Figs. 3 and 4). Only 8 cases showed positive CEA staining over whole cyto- 
plasm of tumor cells (Fig. 5).

Both endocervical and endometrioid type of endocervical adenocarcinomas revealed positive CEA staining over whole cytoplasm more frequently than on apical surface. That is to say, the pattern of CEA distribution in endometrioid type of endocervical adenocarcinoma was not similar to that in primary endometrial adenocarcinoma.

After all, CEA was immunohistochemically detected on whole cytoplasm in most of endocervical adenocarcinomas and on apical surface in most of endometrial adenocarcinomas. This result was statistically significant (chi-square test, $p<0.001)$.

\section{Discussion}

It is sometimes difficult to establish differential diagnosis on endocervical and endometrial adenocarcinomas in routine histological specimens. Several authors reported that these two types of uterine adenocarcinomas could be differentiated by immunoperoxidase technique.

Wahlström et al. (1979) suggested that $80 \%$ of endocervical adenocarcinomas show positive CEA immunostain, while all endometrial adenocarcinomas but adenosquamous carcinomas are CEA negative. In several other papers, positive rate for CEA by immunoperoxidase in cervical adenocarcinoma was reported to be $92-100 \%$ (Cohen et al. 1982 ; Ueda et al. 1983 ; Speers et al. 1983), while it varies from 8 to $52 \%$ in endometrial adenocarcinoma (Goldenberg et al. 1978; Cohen et al. 1982 ; Ueda et al. 1983; Okudaira et al. 1984).

In the present study, $96 \%$ of endocervical adenocarcinomas showed positive CEA staining in agreement with the results reported in other series. Wahlström et al. (1979) reported that all of their 11 cases of endocervical clear cell adenocarcinoma were CEA negative. In our series, the only one case that did not show CEA staining in endocervical adenocarcinomas was clear cell adenocarcinoma. These results suggest that clear cell and pure endocervical adenocarcinoma may have different origin.

On the other hand, positive rate for CEA immunostain in endometrial adenocarcinomas in our series was considerably higher than in the previous reports. In addition, the distribution of positive CEA immunostain was quite different between endocervical and endometrial adenocarcinomas in our study. CEA was stained over whole cytoplasm in most of endocervical adenocarcinomas and on apical surface in most of endometrial adenocarcinomas, respectively. We used $\mathrm{ABC}$ method, which is more sensitive than the conventional immunoperoxidase technique such as PAP method. So, it is supposed that this sensitive method visualized the trace amount of CEA deposited on apical surface, raising the positive rate for CEA staining in endometrial adenocarcinomas. Therefore, the difference of CEA distribution rather than simple CEA positivity might be more useful to distinguish endocervical adenocarcinoma from endometrial one. 
Endocervical adenocarcinoma of endometrioid type is said to develop from ectopic endometrial glands within the cervix (Noda et al. 1983). But in this study, the endocervical adenocarcinoma of endometrioid type and the primary endometrial adenocarcinoma showed different distribution in CEA staining. From these results, it is supposed that their functional characteristics are different.

\section{References}

1) Cohen, C., Shulman, G. \& Budgeon, L.R. (1982) Endocervical and endometrial adenocarcinoma: An immunoperoxidase and histochemical study. Amer. J. Surg. Pathol., 6, 151-157.

2) Gold, P. \& Freedman, S.O. (1965) Demonstration of tumor-specific antigens in human colonic carcinomata by immunological tolerance and absorption techniques. J. exp. Med., 121, 439-462.

3) Goldenberg, D.M., Sharkey, R.M. \& Primus, F.J. (1978) Immunocytochemical detection of carcinoembryonic antigen in conventional histopathology specimens. Cancer, 42, 1546-1553.

4) Hautzer, N.W., Wittkuhn, J.F. \& McCaughey, W.T.E.(1980) Trypsin digestion in immunoperoxidase staining. J. Histochem. Cytochem., 28, 52-53.

5) Hsu, S.M., Raine, L. \& Fanger, H. (1981) Use of avidin-biotin-peroxidase complex $(\mathrm{ABC})$ in immunoperoxidase techniques : A comparison between $\mathrm{ABC}$ and unlabeled antibody (PAP) procedures. J. Histochem. Cytochem., 29, 577-580.

6) Noda, K., Kimura, K., Ikeda, M. \& Teshima, K. (1983) Studies on the histogenesis of cervical adenocarcinoma. Int. J. Gynecol. Pathol., 1, 336-346.

7) Okudaira, Y., Ohtsuru, Y., Matsui, Y., Sawada, M., Ozaki, M., Hongo, J., Hirota, Y., Yamazaki, M. \& Inagaki, M. (1984) Immunohistochemical demonstration of carcinoembryonic antigen and ultrastructural features of the endometrial adenocarcinoma. Acta obst. gynaec. jap., 36, 173-181. (in Japanese with English abstract)

8) Speers, W.C., Picaso, L.G. \& Silverberg, S.G. (1983) Immunohistochemical localization of carcinoembryonic antigen in microglandular hyperplasia and adenocarcinoma of the cervix. Amer. J. clin. Path., 79, 105-107.

9) Ueda, S., Tsubura, A., Izumi, H., Sasaki, M. \& Morii, S. (1983) Immunohistochemical studies on carcinoembryonic antigen in adenocarcinomas of the uterus. Acta path. jap., 33, 59-69.

10) Wagener, C., Csaszar, H., Totovic, V. \& Breuer, H. (1978) A highly sensitive method for the demonstration of carcinoembryonic antigen in normal and neoplastic colonic tissue. Histochemistry, 58, 1-11.

11) Wahlström, T., Lindgren, J., Korhonen, M. \& Seppälä, M. (1979) Distinction between endocervical and endometrial adenocarcinoma with immunoperoxidase staining of carcinoembryonic antigen in routine histological tissue specimens. Lancet, 2, 11591160. 